

\title{
USOS Y EFECTOS DE LAS BANDAS ELÁSTICAS SOBRE LAS CUALIDADES FÍSICAS. REVISIÓN
}

\section{Uses and effects of elastic bands on physical qualities. Review}

Fecha de recepción: 2 de noviembre de 2014 - Fecha de aprobación: 1 de diciembre de 2014

RESUMEN

Las bandas elásticas han sido empleadas para la intervención sobre fuerza, sin embargo, Liu y Latham (2009) no se ha consolidado información sobre usos y efectos sobre otras cualidades. Se propuso documentar los usos y efectos de las bandas elásticas, como un soporte teórico para favorecer la toma de decisiones en intervenciones fisioterapéuticas. Esta investigación se desarrolló como un estudio descriptivo tipo revisión documental; se tomaron 70 artículos de bases de datos como Pubmed, Cochrane y revistas como Physical Therapy, de los cuales 42 cumplieron los criterios de inclusión. El 35\% y 26\% de los artículos correspondió a Ensayos clínicos aleatorizados y no aleatorizados respectivamente, un $5 \%$ correspondió a revisiones sistemáticas o metaanálisis. Aunque los elásticos son empleados principalmente para intervención sobre fuerza ( $40 \%$ de los usos documentados), se evidenciaron otros usos como balance, estabilidad y equilibrio con un $16 \%$, y flexibilidad con un $15 \%$, seguidos en menor proporción por intervención sobre postura, motricidad gruesa y fina, propiocepción, marcha, calidad de vida, y dolor. En cuanto a los efectos, los resultados más consistentes son en fuerza y en flexibilidad, con importantes resultados a favor del tratamiento ( $72 \%$ y $83 \%$ respectivamente). Las demás intervenciones no evidenciaron diferencias significativas a favor del tratamiento con elásticos. Está bien documentada la efectividad de las bandas elásticas sobre la fuerza muscular y la flexibilidad. Los elásticos no son empleados como único tratamiento, lo que dificulta una atribución de efectos específica hacia las bandas. Falta investigación sobre la utilización de bandas elásticas para propiocepción, balance, equilibrio y coordinación.

PALABRAS CLAVE

Fisioterapia, entrenamiento de resistencia, fuerza muscular, bandas elásticas, cualidades físicas.

\begin{abstract}
Elastic bands have been used for intervention in muscle strenght. However, there is not consolidated information about uses and effects in other physical qualities. The main aim of the study is to determine the uses and effects of elastic bands in order to provide theoretical support for decision making in physiotherapy interventions. This research was conducted as a descriptive documental review. Seventy articles were downloaded of databases such as Pubmed, Cochrane and magazines such as Physical Therapy; only 42 met the inclusion criteria. A $35 \%$ and $26 \%$ of articles correspond to randomized and not randomized clinical trials respectively; a $5 \%$ was systematic reviews or metaanalisys. Although the elastic are used primarily for strengt intervention ( $40 \%$ of documented uses), other uses such as balance, stability and balance with $16 \%$, and flexibility with $15 \%$ was evidenced; followed in smaller proportion by intervention posture, gross and fine motor skills, proprioception, walking, quality of life, and pain. In terms of effects, the results are consistent in strength and flexibility, with significant results in favor of treatment $(72 \%$ and $83 \%$ respectively). Other interventions did not show significant differences in favor of treatment with elastic bands. It is well documented the effectiveness of the elastic bands on muscle strength and flexibility. The elastic are not used as a single treatment, which makes difficult the attribution of specific effects directly to the bands. Is necessary more research about use of elastic bands for proprioception, balance, and coordination.
\end{abstract}

KEY WORDS

Physical therapy specialty, resistance training, muscle strength, elastic bands, physical qualities. 
tipo de revisiones tienen como común denominador la aplicación

as cualidades físicas han sido clasificadas como capacidades motrices o físicomotrices. En este sentido, por cuestiones de aprendizaje, se han subdividido además en capacidades condicionales, capacidades coordinativas, y capacidades resultantes; las primeras en función de la energía y el metabolismo necesario para realizarlas (Fuerza, resistencia, velocidad y flexibilidad), las segundas en función de los procesos de control e integración necesarios para su desarrollo (orientación, equilibrio, reacción, ritmo, anticipación, y coordinación) (Universidad de Antioquia, sf); y las terceras como un resultante de la combinación de las dos primeras (López Chicharro, 2001).

Las resistencias progresivas por su principio de acción, han sido empleadas en el manejo de fuerza muscular, sin embargo, esta cualidad fisiológicamente impacta otras cualidades que requieren integridad muscular, por tanto es adecuado pensar que el trabajo muscular puede impactar indirectamente otras cualidades físicas como velocidad, resistencia, coordinación, equilibrio, etc. Ahora bien, la investigación sobre bandas elásticas tiene varias décadas de producción, lo que se traduce en un volumen importante de estudios, muchos de ellos centrados en condiciones específicas de salud.

A partir de esta premisa, se planteó como objetivo de investigación el identificar las características del uso de las bandas elásticas en la intervención de cualidades físicas, reportados en estudios publicados en la literatura científica. Para cumplir este objetivo, se propusieron como objetivos específicos: a) Reconocer en función de las cualidades físicas intervenidas, los usos tradicionales de las bandas elásticas, reportados en estudios publicados, b) reconocer en función de las cualidades físicas intervenidas los usos infrecuentes de las bandas elásticas, reportados en estudios publicados, y c) identificar los efectos sobre las cualidades físicas obtenidos mediante el uso de las bandas elásticas.

Así mismo, a partir de los resultados acá documentados, se podrán generar proyectos aplicados que permitan desde la Fisioterapia abordar poblaciones con diversas condiciones de salud, sugiriendo el trabajo con bandas elásticas como una alternativa para aquellas personas que por sus características particulares, o por las condiciones de los servicios de salud, no puedan ser intervenidas con estrategias tradicionales para cada cualidad física. Por otro lado, una documentación que toma herramientas de las revisiones sistemáticas permite más allá de una búsqueda exhaustiva, realizar una evaluación sobre la calidad de la producción, que se traduce en una herramienta valiosa para la toma de decisiones en la medida en que relaciona los hallazgos de aquellos estudios que contaron con un adecuado proceso investigativo e identifica las investigaciones que metodológicamente presentaron dificultades.

Las bandas elásticas como estrategia terapéutica han sido ampliamente utilizadas alrededor del mundo. Son numerosas las investigaciones realizadas alrededor de la utilidad terapéutica e incluso se encuentran revisiones sistemáticas como la desarrollada por Liu y Latham (2009), que comparó tres tipos de estrategias para la aplicación de resistencia progresiva, incluidas bandas elásticas. Este con fines de entrenamiento en la fuerza y resistencia muscular. Para ampliar la búsqueda de antecedentes sobre investigación alrededor de Thera-Band, se empleó como palabras clave "Elastic band", encontrando diversos ensayos clínicos empleando las bandas elásticas con fines de fortalecimiento, sin embargo, estudios como el de Stevenson, Warpeha, Dietz, Giveans y Erdman (2010), proponen los efectos de las bandas elásticas también en la velocidad, lo que sumado al hecho que los patrones de movimiento ejecutados para los trabajos activos implican el desarrollo de otras cualidades como flexibilidad y propiocepción, sugiere que las bandas elásticas podrían tener usos más allá de la fuerza y la resistencia, por tanto este proceso de revisión detallada de la investigación publicada, permite hacer evidentes los diversos usos terapéuticos de las bandas elásticas, y se constituye en un punto de partida para futuros desarrollos investigativos aplicados, fortalece las bases de prescripción no sólo para el trabajo de fuerza muscular, sino de otras cualidades físicas que directa e indirectamente se pueden desarrollar y mantener a partir del trabajo con resistencias progresivas.

Las bandas elásticas surgieron como una idea innovadora de la empresa Hygienic Dental Rubber Company; una empresa encargada de elaborar elementos para odontología y expertos en el uso de caucho y látex. En los años 80, la empresa desarrolló las bandas como una forma de diversificar sus productos más allá de lo odontológico; las bandas fueron llamadas Theraband ${ }^{\circledR}$, siendo de utilidad para el entrenamiento deportivo, y más adelante fueron incorporadas por Fisioterapeutas para las áreas de la rehabilitación (Theraband, Systems of progresive excercise, sg).

\section{MÉTODO}

Esta investigación se desarrolló como un estudio descriptivo de revisión documental que permitió caracterizar la intervención fisioterapéutica con bandas elásticas reportada en la literatura. La investigación descriptiva hace evidentes características de personas, objetos, situaciones o fenómenos de interés (Dankhe, citado por Hernández, Fernández y Baptista, 2012), lo que permite en este contexto identificar las características de la intervención con bandas elásticas y favorece la delimitación de un punto de partida para futuros programas de intervención de cualidades físicas que puedan emplear dichas bandas como herramienta central tanto para el manejo de personas sanas como con patología en diferentes contextos. La investigación se realizó bajo un diseño tipo revisión documental, que permite "reunir toda la evidencia empírica [...] con el fin de responder una pregunta específica de investigación" (Centro Cochrane Iberoamericano, 2011).

La investigación documentó la producción investigativa analizando tanto la calidad metodológica de las investigaciones revisadas como aspectos conceptuales sobre usos y los efectos logrados a partir del uso de las bandas elásticas. Se realizó una búsqueda exhaustiva que permitió documentar la producción específicamente desde estudios de intervención, sin embargo, se incluyeron investigaciones observacionales en la medida en que los hallazgos desde ensayos clínicos aleatorizados y controlados fueron limitados para documentar usos de las bandas. 
La investigación tomó fuentes primarias de información, específicamente estudios de intervención, sin embargo en función de los hallazgos, se dio lugar al registro y análisis de otros diseños de la siguiente forma (ver tabla 1):

Tabla 1. Selección de las fuentes para la revisión.

\begin{tabular}{|c|c|c|}
\hline Búsqueda & Diseño de investigación & Alcance \\
\hline Primaria & $\begin{array}{l}\text { Revisiones sistemáticas / Ensayo clínico } \\
\text { aleatorizado y controlado }\end{array}$ & \multirow{3}{*}{ Intervención } \\
\hline Secundaria & Ensayos clínicos aleatorizados & \\
\hline Terciaria & $\begin{array}{l}\text { Ensayos clínicos no aleatorizados, no } \\
\text { controlados }\end{array}$ & \\
\hline Cuaternaria & Reportes de caso & Observaciona \\
\hline
\end{tabular}

La búsqueda se realizó inicialmente por medio de metabuscadores, empleando combinaciones de palabras clave como: [Bandas elásticas]; [Theraband]; [Ensayo clínico]; [intervención]; [tratamiento]; [resistencia progresiva]; [cualidad física]; [fuerza]; [velocidad]; [resistencia]; [coordinación]; [equilibrio]. A partir de la búsqueda inicial se identificaron los términos MeSH (DeCS) a partir de los cuales se realizaron búsquedas específicas en bases de datos como Medline, Cochrane y Bireme. Finalmente se realizó búsqueda específica número a número en la revista Physical Therapy, como principal publicación internacional.

Para la extracción y sistematización de la información se desarrolló una ficha de registro que incluyó elementos como: a) Fuente: Información del estudio, información del revisor, referencia y detalles de contacto; b) elegibilidad: Criterios de elegibilidad para la revisión, motivo de exclusión, c) método documentado por el estudio; d) participantes: Número total, ámbito, fecha del estudio; e) Uso específico, detalles de la intervención; f) Efectos (desenlaces); y g) resultados. Por su alcance documental, los recaudos éticos en esta investigación se relacionarán con el cuidado en el manejo de la información y los conflictos de interés; así, dado que se recomienda que las revisiones sean realizadas evitando intereses comerciales, para efecto de la presente investigación se buscó que la empresa comercial que tradicionalmente ha producido las bandas elásticas no intervenga en la interpretación de ningún resultado; así se garantiza que la Escuela Colombiana de Rehabilitación no tiene ninguna intención comercial que pueda impactar los resultados de esta investigación.

En cuanto a las fuentes consultadas, se realizó un registro sistemático, respetando los procesos de citación y referenciación, de modo que se respete la autoría correspondiente a cada autor consultado.

Para el desarrollo de la investigación se diseñó una planilla de registro de artículos que permitió identificar aquellos estudios que cumplen con los criterios de inclusión, de modo que la revisión de calidad metodológica se realizó sólo a aquellos estudios con potencial de aporte a los resultados.

Se realizó la búsqueda de información relacionada con el uso y los efectos de las bandas elásticas Thera-band ${ }^{\circledR}$ reportados en la literatura científica. Para esto se recurrió a diversos tipos de búsque- da: inicialmente se realizó una búsqueda abierta en metabuscador "scholar.google.com", para la cual se empleó una combinación de palabras clave como "Elastic", "bandas elásticas", "physiotherapy", "thera-band ${ }^{\circledR}$ ", "progressive Ressistance"; o combinaciones de ellas. Esta búsqueda abierta permitió identificar investigaciones que de alguna forma nombraban el uso de bandas elásticas, pero no necesariamente como tratamiento principal, y no necesariamente permitían la identificación de desenlaces impactados por el uso de herramientas elásticas de resistencia progresiva. A partir de esto, se modificó la estrategia de búsqueda desde dos vías: en primer lugar se realizó una búsqueda seleccionando una publicación representativa de la investigación en Fisioterapia, en este caso el "Physical Therapy Journal”, esta publicación fue revisada empleando como palabras clave las mismas de la búsqueda abierta. En segundo lugar se realizó la búsqueda en la página de "The Thera-band ${ }^{\circledR}$ academy" empleando la estrategia de búsqueda de la página, especificando por producto “Thera-Band ${ }^{\circledR}$ Elastic resistance”.

Dado que cuando se emplea como fuente de información el fabricante o proveedor de un producto se cae en el riesgo de sesgos de publicación, por tanto, durante la revisión en la página de Thera-band ${ }^{\circledR}$ se realizó un registro de las publicaciones de modo que no se privilegiara una publicación en especial. Aunque esta estrategia no elimina la posibilidad de sesgo de publicación, genera un primer filtro que luego se complementa con los análisis de contenido y el análisis de conflictos de interés que sean manifestados en las investigaciones que son incluidas en la investigación.

\section{RESULTADOS}

Un primer hallazgo de importancia al emplear la estrategia de búsqueda en la página web de la Thera-band ${ }^{\circledR}$ academy es que tiene clasificados los resultados de investigación por tipos de intervención, lo que necesariamente impacta los resultados de esta investigación por cuanto se vislumbra que el fabricante tiene claridad sobre los múltiples impactos de la estrategia de intervención más allá del entrenamiento de fuerza. Las áreas que documenta la Thera-band academy, fueron: a) balance / estabilidad, b) cardiorespiratorio / aeróbico, c) entrenamiento funcional, d) marcha, e) movilidad / rango de movimiento, f) postura, g) entrenamiento de potencia, h) propiocepción, i) entrenamiento neuromuscular, j) resistencia / entrenamiento de fuerza, k) entrenamiento sensoriomotriz, 1) movilización de tejido blando, $\mathrm{m}$ ) velocidad y agilidad,n) entrenamiento de estabilidad, y o) estiramientos / flexibilidad.

\section{Desenlaces}

Para la propuesta de desenlaces se buscó abrir una puerta amplia de forma que en consecuencia con el alcance de la investigación se lograran documentar la mayor cantidad de usos potenciales de las bandas elásticas, lo cual se refleja en las variables que son impactadas. En este sentido, se emplearon las variables contempladas por los desarrolladores de las bandas Thera-band ${ }^{\circledR}$, haciendo claridad que al inicio de la búsqueda no se esperó que todos los desenlaces tuvieran resultados asociados a las bandas elásticas. De esta forma, los desenlaces propuestos para el análisis son: 
Actividades de la vida diaria, adherencia / distensibilidad, agilidad, balance / estabilidad, comportamiento, el flujo sanguíneo, lípidos en sangre / glucosa, presión sanguínea, composición corporal, densidad mineral ósea, gasto calórico, función cardiovascular, coordinación, costo efectividad, discapacidad, estado de la enfermedad, respuesta a la dosis, edema, caídas / riesgo de caídas, fatiga, flexibilidad / ROM, rendimiento funcional / físico, capacidad funcional, marcha y locomoción, fuerza de agarre, estado de salud, hormonas, función Inmune, respuesta inflamatoria, tasa de lesiones, movilidad e integridad articular, distancia / altura de salto, cinética / cinemática, gasto mental/cognitivo o psicológico, gasto metabólico / calorías, metabolismo, movilidad, función motora, activación muscular (EMG), masa muscular, resistencia muscular, fuerza muscular, resistencia muscular, dolor, esfuerzo percibido,nivel de actividad física, postura, propiocepción, calidad de vida, reducción y prevención de riesgos, seguridad, autoestima, sensación, velocidad, estabilización / estabilidad articular.

Emplear the Thera-band ${ }^{\circledR}$ academy como fuente implica una amplia fuente de información que asegura artículos relacionados con bandas elásticas, sin embargo requiere una mirada más cuidadosa a los posibles conflictos de interés, de modo que se analizará con más detenimiento. Los hallazgos de artículos en la plataforma de Thera-band ${ }^{\circledR}$ bajo las palabras clave de interés arrojó un total de 1559 posibles artículos a analizar, lo cual se aleja de las posibilidades de análisis de esta investigación. A razón de ello se realizó un proceso de selección intencional, con base en las proporciones de artículos disponibles por área de intervención.

Se recuperaron artículos bajo 3 estrategias: de búsqueda a) búsqueda libre por palabra clave, b) búsqueda en el Physycal Therapy Journal, y c) búsqueda por temática en la página web de The Thera-band ${ }^{\circledast}$ Academy.

Al realizar la revisión de los artículos, algunos no tenían referencias precisas al uso de bandas elásticas, por cuanto para el análisis final, se contó con 42 artículos que fueron finalmente analizados, de esta forma, se realizará una delimitación de los usos y efectos documentados por las investigaciones.

\section{Características de los artículos revisados}

La figura 1 muestra la distribución de los artículos incluidos en el análisis, según el año de publicación. Es evidente la relación de 5 (cinco) artículos con un periodo de publicación mayor a 10 años, sin embargo, para efectos de los usos y efectos de las bandas elásticas (Thera-band ${ }^{\circledR}$ ), la temporalidad de los estudios no afecta los resultados. La mayor concentración de investigaciones se encontró entre 2009 y 2013 , con 27 documentos.

Se planteó como criterio de inclusión la pertinencia de ensayos clínicos principalmente, sin embargo, dada la limitación en el acceso a estudios de alcance explicativo, se amplió la mirada a aquellos estudios con alcances más limitados, pero que dan cuenta de los usos y efectos potenciales asociados a las bandas elásticas. La descripción de los tipos y diseños de investigación son presentados en la figura 2, y hacen evidente la prioridad hacia ensayos clínicos, pero además hacen evidente un número aceptable de estudios descriptivos con diferentes métodos/diseños.

\section{Usos y efectos de las bandas elásticas / Thera-band ${ }^{\circledR}$}

Ahora bien, uno de los elementos claves del estudio es la identificación de los usos de las Thera-band ${ }^{\circledR}$, en este sentido, como es de esperar por la tradición en el uso de las bandas elásticas, se encontró una tendencia importante de artículos que emplearon las Thera-band ${ }^{\circledR}$ para entrenamiento de fuerza, sin embargo, se encuentran además otros usos que no son de frecuente aparición como balance, estabilidad y equilibrio y flexibilidad (ver figura 3). Diversos estudios mostraron otros usos como marcha, balance, estabilidad y equilibrio o dolor (entre otros usos); el uso central era la fuerza como medio para lograr impactar otras cualidades. Un ejemplo es

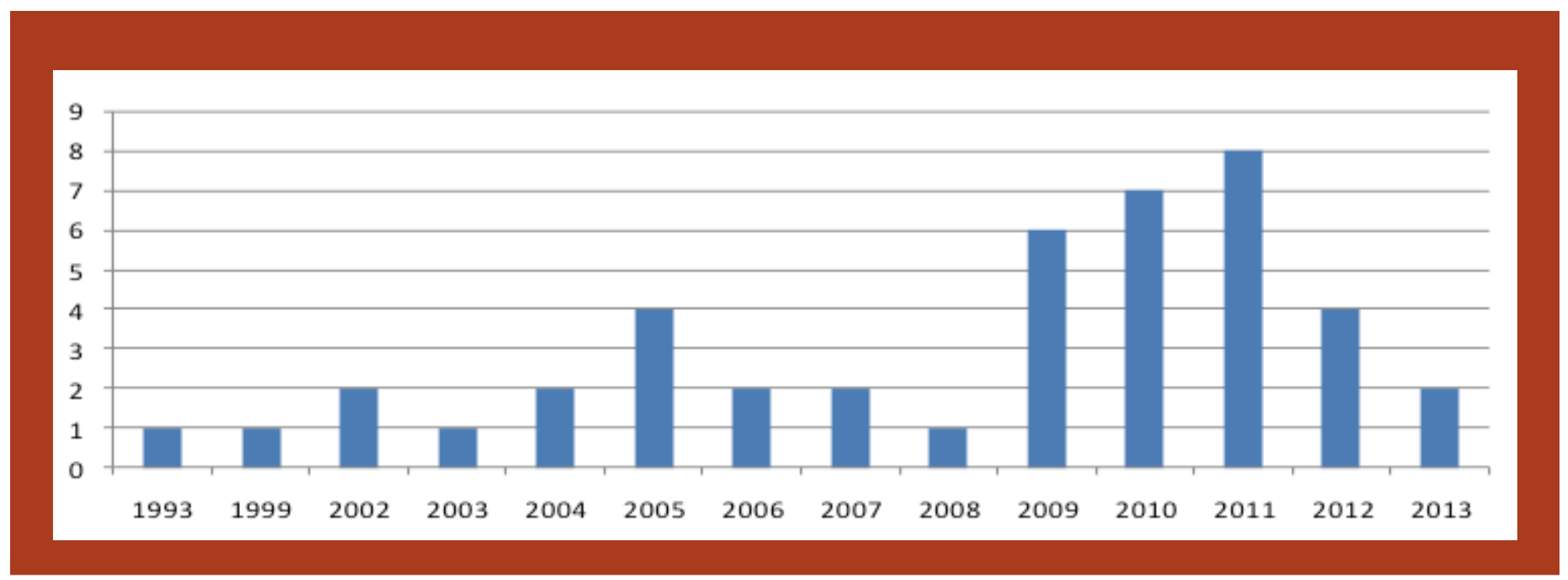

Figura 1. Años de publicación de los artículos reportados en la investigación. 


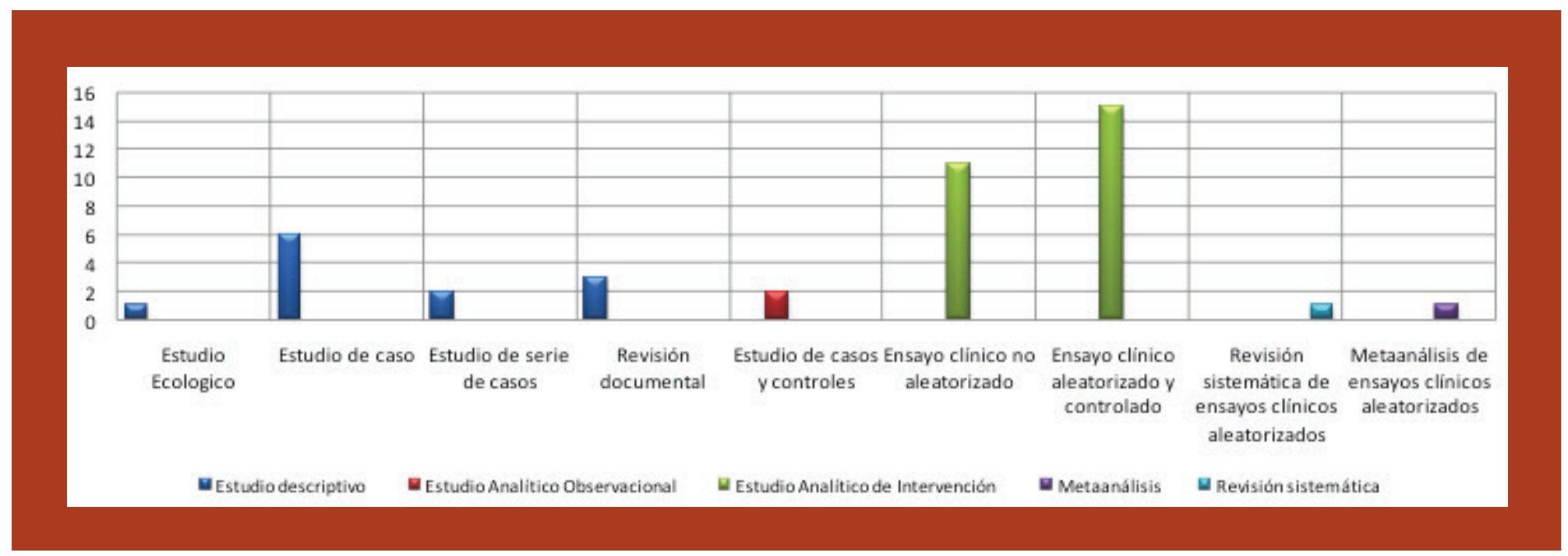

Figura 2. Tipos de estudios y diseños incluidos.

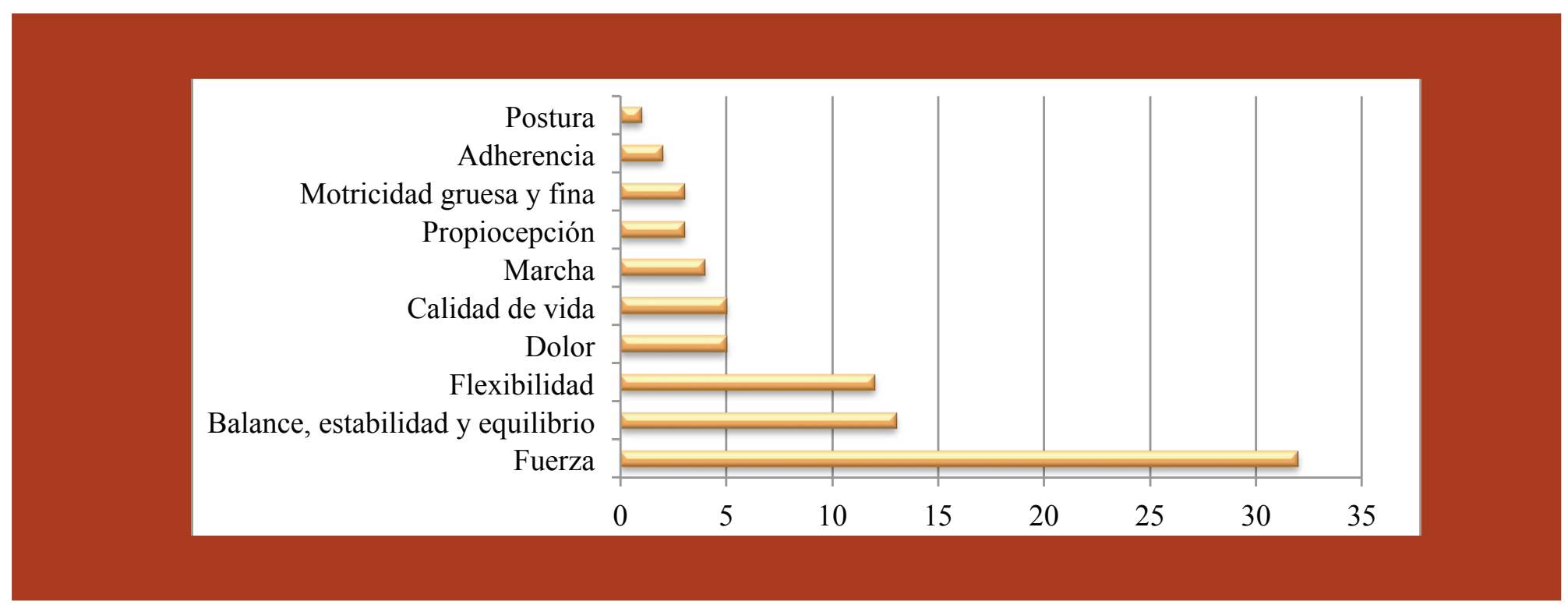

Figura 3. Usos encontrados en los artículos recuperados.

el estudio de Van Ettekoven y Lucas (2006), quienes impactaron el dolor asociado a cefalea tensional, a partir del trabajo de fortalecimiento cervical como un determinante de la estabilidad de columna cervical, que indirectamente favorece una reducción en el dolor.

Ahora bien, se debe precisar que sólo dos artículos, emplearon exclusivamente Thera-Band ${ }^{\circledR}$ como herramienta de intervención, por tanto los resultados que se presentan a continuación, no pueden ser atribuibles exclusivamente a las bandas elásticas.

Los resultados se presentarán en 5 categorías en función del nivel de evidencia, desde mayor nivel a menor nivel: a) Revisión sistemática, b) Ensayo clínico aleatorizado y controlado, c) ensayo clínico no aleatorizado, d) estudios de caso y series de caso, y e) estudio documental.
Crandall, Howlett y Keysur (2013) publicaron el estudio "Exercise Adherence Interventions for Adults With Chronic Musculoskeletal Pain”, la investigación correspondió a una revisión sistemática de ensayos clínicos aleatorizados en donde se trabajó con ejercicio aeróbico, ejercicio de resistencia, hidroterapia, tai chi, ejercicios isocinéticos, ejercicios de estabilización, bicicleta, flexibilidad, yoga y ejercicios de reeducación postural para disminuir el dolor, se encontró que no hay diferencias estadísticamente significativas entre las intervenciones pero se aclara que todas disminuyeron el dolor en los grupos experimentales.

Son múltiples las investigaciones experimentales que evidencian resultados a favor de la aplicación de bandas elásticas en conjunto con tratamientos tradicionales. Los resultados asociados a las inves- 
12 tigaciones tipo ensayo clínico, estudios de caso, estudios de cohorte, estudios de casos y controles, y estudios documentales son presentados en el las tablas 2 a 5 .

Tabla 2. Resultados de los ensayos clinicos aleatorizados.
Por otro lado, se realizó un análisis de los hallazgos en función de los efectos obtenidos diferenciados según el diseño de investigación. Teniendo en cuenta que no todas las investigaciones repor-

\begin{tabular}{|c|c|c|}
\hline \multicolumn{3}{|c|}{ Ensayo clínico aleatorizado y controlado. } \\
\hline Cualidad impactada & Resultado de la investigación & Autor \\
\hline Fuerza muscular y flexibilidad & A favor del grupo experimental (bandas elásticas) & Kemmler, Lauber, Weineck, Hensen, Kalender y Engelke (2006) \\
\hline Fuerza muscular & A favor del grupo experimental (bandas elásticas) & Page, Lamberth, Abadie, Boling, Collins y Linton (1993) \\
\hline Fuerza muscular, el balance y el equilibrio & A favor del grupo experimental (bandas elásticas) & $\begin{array}{l}\text { Carter, Khan, McKay, Petit, Waterman, Heinonen, Janssen, } \\
\text { Donaldson, Mallinson, Riddell, Kruse, Prior y Flicker (2002) }\end{array}$ \\
\hline Fuerza muscular. & $\begin{array}{l}\text { No hubo diferencias estadisticamente significativas. } \\
\text { Las dos intervenciones mejoraron la cualidad. }\end{array}$ & Fitzgeraid, Piva, Gil, Wlsnnelwski, Oddis y Irragang (2011) \\
\hline Fuerza muscular + balance. & $\begin{array}{l}\text { A favor del grupo experimental (bandas elásticas) } \\
\text { sólo para una de las pruebas realizadas }\end{array}$ & Szturm, Betker, Moussavi, Desai, y Goodman (2011) \\
\hline Dolor y marcha & A favor del grupo experimental (bandas elásticas) & Piva, Gil, Almeida, DiGioia III, Levison y Fitzgerald (2010) \\
\hline Dolor y adherencia al tratamiento & No hubo diferencias estadísticamente significativas. & $\begin{array}{l}\text { Andersen, Hannerz, Saervoll, Zebis, Mortensen, y } \\
\text { Poulsen (2011) }\end{array}$ \\
\hline Fuerza muscular y balance & No hubo diferencias estadísticamente significativas. & Unsgaard-Tøndel, Fladmark, Salvesen y Vasseljen (2014) \\
\hline $\begin{array}{l}\text { Flexibilidad, fuerza de resistencia, } \\
\text { actividades de la vida diaria, y relajación. }\end{array}$ & No hubo diferencias estadísticamente significativas. & $\begin{array}{l}\text { Inge, Van Haren, Timmermant, Potting, Blijle Vens, Slaal y } \\
\text { Huis -Vander Sanden (2013) }\end{array}$ \\
\hline Fuerza de resistencia & $\begin{array}{l}\text { A favor del grupo experimental (bandas elásticas), } \\
\text { con limitación por pobre análisis estadístico. }\end{array}$ & Topp, Pague, Swank, Quesada, Nyland, y Malkani (2009) \\
\hline Fuerza & $\begin{array}{l}\text { A favor del grupo experimental (bandas elásticas), } \\
\text { con limitación por pobre análisis estadístico. }\end{array}$ & $\begin{array}{l}\text { Swank, Kachelman, Bibeau, Quesada, Nyland, Malkani, y } \\
\text { Toop, (2011) }\end{array}$ \\
\hline Fuerza, flexibilidad y velocidad en deporte & A favor del grupo experimental (bandas elásticas) & $\begin{array}{l}\text { Fernandez-Fernandez, Ellenbecker, Sanz-Rivas, Ulbricht } \\
\text { y Ferrauti, (2013) }\end{array}$ \\
\hline Postura, dolor, flexibilidad & $\begin{array}{l}\text { A favor del grupo experimental (bandas elásticas). } \\
\text { Las dos intervenciones causaron mejoría. }\end{array}$ & Van Ettekoven, y Lucas, (2006) \\
\hline Fuerza muscular & A favor del grupo experimental (bandas elásticas) & Nilsson, Jonsson y Ekdahl, (2009) \\
\hline Fuerza muscular y propiocepción & $\begin{array}{l}\text { No hubo diferencias estadísticamente significativas. } \\
\text { Las dos intervenciones generaron efecto. }\end{array}$ & Smith, Docherty, Simon y Klossner, Schrader (2012) \\
\hline Fuerza y flexibilidad & No hubo diferencias estadísticamente significativas. & Hale, Hertel y Olmsted-Kramer (2007) \\
\hline Balance, control y propiocepción & No hubo diferencias estadísticamente significativas. & Myer, Ford, Palumbo y Hewitt (2005) \\
\hline Fuerza muscular empleando Thera-tube ${ }^{\circledR}$ & A favor del grupo experimental (bandas elásticas) & Johansson, Adolfsson, y Foldevi (2005) \\
\hline Postura y la densidad ósea & No hubo diferencias estadísticamente significativas. & $\begin{array}{l}\text { Preisinger, Alacamlioglu, Pils, Bosina, Metka, Schneider y } \\
\text { Ernst (2011) }\end{array}$ \\
\hline
\end{tabular}

Tabla 3. Resultados de los ensayos clìnicos no aleatorizados, cohorte y casos y controles.

\begin{tabular}{|c|c|c|}
\hline \multicolumn{3}{|c|}{ Ensayo clínico controlado no aleatorizado } \\
\hline Cualidad impactada & Resultado de la investigación & Autor \\
\hline Fuerza muscular & A favor del grupo experimental (bandas elásticas) & Witt, Talbott, Kotowski (2011) \\
\hline Fuerza muscular y marcha & No hubo diferencias estadísticamente significativas. & English, Hillier, Stiller y Warden-Flood (2007) \\
\hline Fuerza muscular & $\begin{array}{l}\text { No hubo diferencias estadísticamente significativas, } \\
\text { salvo en aducción horizontal. }\end{array}$ & Wang, McClure, Pratt, y Nobilini, (1999) \\
\hline Fuerza de resistencia & No hubo diferencias estadisticamente significativas. & Jacobs, Lewis, Bolgla, Chistersen, Nitz, y Uhl, (2009) \\
\hline Fuerza muscular y flexibilidad & No hubo diferencias estadísticamente significativas. & Bellew, Frilot, Busch, Tong, Lomothe, and Ozane (2010) \\
\hline Fuerza muscular y flexibilidad & No hubo diferencias estadísticamente significativas. & $\begin{array}{l}\text { Conti, Garafolo, Dele Rose, Massazza, Venci, Randelli, y } \\
\text { Castagna, (2009) }\end{array}$ \\
\hline \multicolumn{3}{|c|}{ Estudio de cohorte } \\
\hline Balance & A favor del grupo experimental (sandalias) & Blackburn; Hirth; Guskiewicz (2003) \\
\hline \multicolumn{3}{|c|}{ Estudio de Casos y controles } \\
\hline Fuerza muscular y esfuerzo percibido & $\begin{array}{l}\text { No hubo diferencias estadísticamente significativas. } \\
\text { Las dos intervenciones generaron efecto. }\end{array}$ & $\begin{array}{l}\text { Andersen, Andersen, Mortensen, Poulsen, Bjørnlund, y } \\
\text { Zebis (2010) }\end{array}$ \\
\hline
\end{tabular}




\begin{tabular}{l|l|l|}
\hline \multicolumn{1}{c|}{ Cualidad impactada } & \multicolumn{1}{c}{ Resultado de la investigación } & \multicolumn{1}{c}{ Autor } \\
\hline Fuerza muscular & No hubo diferencias estadísticamente significativas. & Fragala-Pinckham, Jaley, Rabin, y kharasch (2005) \\
\hline Fuerza muscular & A favor del grupo experimental (bandas elásticas) & Mattacola y Dwyer (2002) \\
\hline $\begin{array}{l}\text { Fuerza muscular, balance, estabilidad y } \\
\text { equilibrio, dolor y calidad de vida. }\end{array}$ & A favor del grupo experimental (bandas elásticas) & Moser (2009) \\
\hline Fuerza muscular y postura & A favor del grupo experimental (bandas elásticas) & Tonley, Yun, Kochevar, Dye, Farrokhi, y Powers, (2010) \\
\hline Fuerza muscular & A favor del grupo experimental (bandas elásticas) & Schmitt, Schmitt, y Rudolph, (2004) \\
\hline Fuerza muscular y flexibilidad & A favor del grupo experimental (bandas elásticas) & Culp and Romani (2006) \\
\hline Fuerza muscular & No hubo diferencias significativas. & Wilcox III, Arslanian, y Millett, (2005) \\
\hline
\end{tabular}

Tabla 5. Resultados de los estudios documentales.

\begin{tabular}{|l|l|l|}
\hline \multicolumn{1}{|c|}{ Cualidad impactada } & \multicolumn{1}{c|}{ Estudios documentales } \\
\hline Fuerza muscular & A favor del grupo experimental (bandas elásticas) & Ellenbecker; Pluim;Vivier; y Sniteman (2009) \\
\hline Fuerza muscular, balance y estabilidad & No hubo diferencias significativas. & Frank, Kovesova y Kolar (2013) \\
\hline
\end{tabular}

tadas tienen un alcance explicativo (experimental), se muestra un resultado que debe ser manejado con cautela, dado que se interpretó como diferencias a favor de la intervención, aquellos estudios que (a pesar de no tener soporte estadístico), manifestaran resultados positivos atribuibles a la intervención. Ahora bien, aunque se encuentra que tanto los ensayos clínicos aleatorizados como no aleatorizados mostraron resultados a favor de la intervención, la revisión sistemática y el metaanálisis encontrado no mostraron resultados diferentes entre las intervenciones (ver figura 4).

Un elemento importante de reportar es la presencia de diferencias obtenidas al realizar la intervención, según la cualidad física o atributo involucrado. En este sentido, se encontró que las mayores diferencias numéricas (no es pertinente realizar interpretaciones inferenciales), se encontraron en el abordaje de fuerza y flexibilidad, sin embargo será pertinente analizar las diferencias en la calidad de vida y en la marcha (ver tabla 6).

Tabla 6. Presencia de diferencias asociadas a la intervención según atributo intervenido.

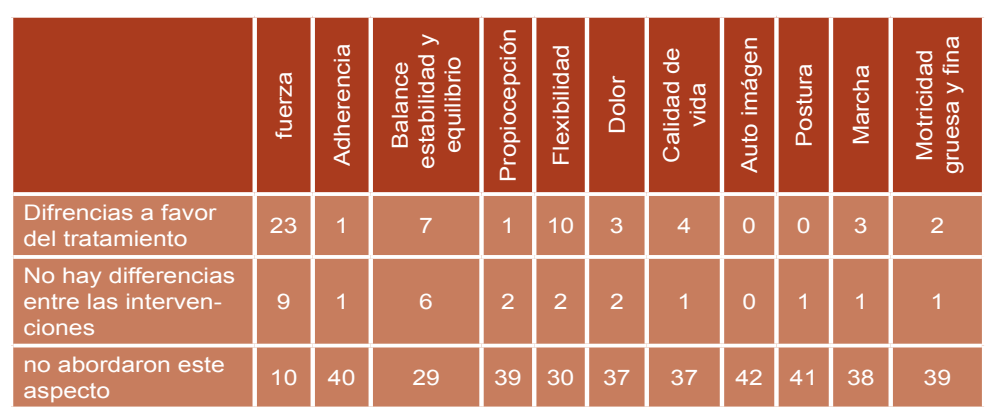

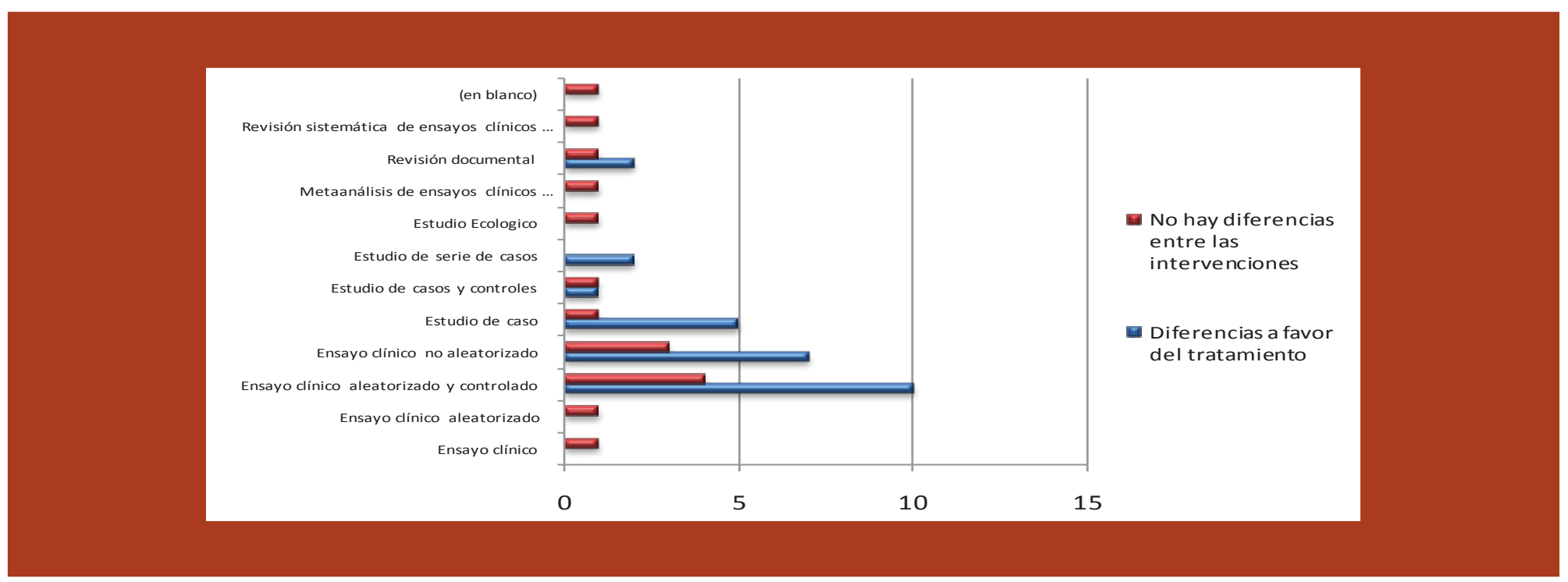

Figura 4. Diferencias en las intervenciones por diseños de investigación. 
Otro hallazgo importante se relacionó con estudios en los cuales

Esta investigación inició bajo la idea de usos extendidos de las bandas elásticas más allá de lo tradicional. La búsqueda llevó a documentos interesantes que reiteradamente citaban a las bandas elásticas como un medio para entrenar fuerza bajo la estrategia de resistencia progresiva (en función de los colores) (The Hygenic Corporation (2006). La búsqueda de información sugería indirectamente el uso de las bandas como una herramienta de entrenamiento y rehabilitación que podía impactar cualidades físicas más allá de la fuerza, así comenzó la búsqueda de información que finalizó con la información presentada.

Los hallazgos permiten hacer diferentes análisis tanto desde lo metodológico, como desde la explicación biológico-fisiológica. En primer lugar, desde los aspectos metodológicos se encuentran varios factores que deben ser analizados para no realizar interpretaciones apresuradas sobre el efecto multifactorial de las bandas elásticas.

La tabla 10 muestra efectos a favor del tratamiento a favor de la técnica en varias cualidades como fuerza, flexibilidad y balance, estabilidad y equilibrio; sin embargo, realizar una apreciación de causa efecto directa, es inadecuado en la medida en que las investigaciones que lo soportan no tuvieron estas cualidades como únicos factores de modificación, y además, las bandas elásticas no fueron empleadas como único medio. Un caso puntual es la investigación de Szturm, Betker, Moussavi, Desai, y Goodman (2011) quienes compararon el efecto de Thera-band + otra intervención, sobre la fuerza, y el balance. Los autores encontraron diferencias a favor del tratamiento experimental sólo para la prueba de parar y seguir (balance y equilibrio), sin embargo el uso de técnicas combinadas no permiten atribuir el efecto específicamente a las bandas elásticas.

El estudio de Van Ettekoven y Lucas, (2006) también incluyó como parte del protocolo enfoques múltiples, en este caso diversas formas de masaje + un programa de entrenamiento cráneo-cervical que utiliza ejercicios de resistencia bajo carga. En este caso, se evidencian factores tanto metodológicos como fisiológicos que deben ser explicados. En primer lugar, la combinación de estrategias (todas dirigidas a la reducción del dolor, lleva a la dificultad de realizar atribuciones causales hacia una sola, de modo que el efecto debe ser documentado como producto de múltiples tratamientos. Así mismo, en segundo lugar los investigadores en este estudio hacen explícito el uso de las bandas elásticas con intención de entrenamiento de fuerza como condicionante del balance osteo-muscular, y éste último como reductor de dolor. Esta descripción es importante, dado que la reducción del dolor no se realiza como efecto directo de las bandas, pero si como un efecto logrado a partir de la estabilización de estructuras desencadenantes de dolor. Así, este tipo de estudios sugiere la prescripción de bandas incluso durante procesos dolorosos que dependan de estabilidad estructural.

Lisón y cols (1998), citados por López Miñaro (sf), mencionan que "diferentes estudios han demostrado la relación entre debilidad y dolor lumbar [...] asimismo, un entrenamiento adecuado de los músculos lumbares puede contribuir a acelerar el proceso de recuperación resultando ser de gran utilidad en el ámbito terapéutico" (p. 4); lo cual refuerza los hallazgos encontrados. no se encontraron diferencias estadísticamente significativas entre los tratamientos, sin embargo, esto lejos de ser un inconveniente, sugiere la equivalencia del uso de bandas elásticas como medio alternativo (y económico) al uso de otros medios de entrenamiento de fuerza de resistencia (con posibilidad de resistencia progresiva). En este sentido, el estudio de Andersen, Hannerz, Saervoll, Zebis, Mortensen, y Poulsen (2011) buscó identificar el efecto de ejercicios de resistencia sobre el dolor cervical, específicamente en cuello y hombros; así como sobre la adherencia al tratamiento. Los autores encontraron como resultado efectos en los dos tratamientos experimentales (Thera-Band ${ }^{\circledR}$ y charlas semanales), versus el grupo de control, sin embargo, no se encontraron diferencias entre los tratamientos.

Otros estudios con resultados similares fueron desarrollados por Andersen, Andersen, Mortensen, Poulsen, Bjørnlund, y Zebis (2010) quienes compararon la aplicación de Thera-Band ${ }^{\circledR}$ (tubos elásticos) Vs ejercicio estándar (mancuernas), sin embargo, no encontraron diferencias en el nivel de fuerza logrado con las intervenciones. Así mismo Fitzgeraid, Piva, Gil, WIsnnelwski, Oddis y Irragang (2011) compararon el uso de Thera-Band ${ }^{\circledR}$ Vs standard sobre la fuerza, el balance y equilibrio sin encontrar diferencias en el efecto logrado entre las intervenciones. Por último, Fragala-Pinckham, Jaley, Rabin, y kharasch (2005) documentaron el efecto de Thera-Band ${ }^{\circledR}+$ otra intervención para el entrenamiento de fuerza de miembros superiores y tronco; sin diferencias entre las intervenciones.

Otro factor importante que se debe analizar, es la diversidad de estudios encontrados, y la falta de mediciones robustas. Como se mencionó al inicio del apartado de resultados, se encontraron múltiples estudios no experimentales que reportaron efectos asociados al uso de las bandas elásticas. Si se evalúan estos resultados al marco de los niveles de evidencia, empleando la clasificación de Oxford (Manterola y Zavando, 2009), estos estudios estarían en un nivel evidencia 4, con un grado de recomendación C, lo cual necesariamente limita la posibilidad de decisión clínica. En la misma línea de análisis se encuentran aquellos ensayos clínicos que presentan análisis estadísticos menos robustos, dado que limitan la posibilidad de inferencia estadística. Tal es el caso de los estudios de Topp, Pague, Swank, Quesada, Nyland, y Malkani (2009); y Swank, Kachelman, Bibeau, Quesada, Nyland, Malkani, y Toop, (2011); quienes investigaron sobre los efectos del entrenamiento pre-quirúrgico, encontrando diferencias a favor de las intervenciones, pero los análisis estadísticos no son rubustos.

Se puede concluir que el uso de bandas elásticas en fisioterapia está bien documentado para diferentes cualidades físicas. A pesar de mantenerse la tendencia de uso hacia el abordaje de la fuerza muscular, se encontró acciones importantes en la flexibilidad, y usos menores en cualidades físicas como sobre postura, motricidad gruesa y fina, propiocepción, marcha, calidad de vida, y dolor. En cuanto a los efectos, aunque se presentan diferencias significativas a favor de las intervenciones que emplearon bandas elásticas, el hecho de no contar con suficientes estudios que hayan empleado exclusivamente bandas elásticas, dificulta una atribución causal plena a favor de los elásticos. Faltan más investigaciones en cualidades físicas en patologías específicas que soporte la utilización de bandas elásticas en dichas condiciones. 


\section{REFERENCIAS}

Andersen, L; Andersen, C.; Mortensen, O.;Poulsen, O.; Bjørnlund, IB. \& Zebis, M. (2010). Muscle Activation and Perceived Loading During Rehabilitation Exercises: Comparison of Dumbbells and Elastic Resistance. Physical Therapy 90:538-549. Recuperado de http//www.apta.org.

Andersen, L.; Hannerz, H.; Saervoll, Ch.; Zebis, M.; Mortensen, O.; y Poulsen O. (2011). Effectiveness of small daily amounts of progressive resistance training for frequent neck/shoulder pain: Randomised controlled trial. Pain. 152 (2011) 440-446. International Association for the Study of Pain. Published by Elsevier B.V. doi:10.1016/j. pain.2010.11.016.

Bellew, Frilot, Busch, Tong, Lomothe, \& Ozane (2010). Facilitating activation of the peroneus longus: electromyographic analysis of exercises consistent with biomechanical function. Clinical trial. Journal of strength and conditioning research.Vol 24 Num 2.

Blackburn; Hirth \& Guskiewicz (2003). Exercise Sandals Increase Lower Extremity Electromyographic Activity During Functional Activities, experimental study type cohort. Journal of athletic training, (3) 198-203.

Carter, Khan, McKay, Petit, Waterman, Heinonen, Janssen, Donaldson, Mallinson, Riddell, Kruse, Prior, Flicker (2002). Community-based exercise program reduces risk factors for falls in 65- to 75-year-old women with osteoporosis: randomized controlled trial. Canadian Medical Association.

Centro Cochrane Iberoamericano, traductores (2011). Manual Cochrane de revisiones sistemáticas de intervenciones, versión 5.1.o. Barcelona. Versión electrónica. Disponible en http://www.cochrane.es/?$\mathrm{q}=\mathrm{es} /$ node $/ 269$.

Conti, M.; Garafolo, R.; Dele Rose, G.; Massazza, G.; Venci, E.; Randelli, M.M \& Castagna, A. (2009). Post-operative rehabilitation after surgical repair of the rotator cuff.Descriptive study. Rescatado de www. musculoskeletalsurg.com.

Crandall, S.; Howlett \& Keysur, J. (2013). Exercise Adherence Interventions for Adults With Chronic Musculoskeletal Pain: systematic review. Physical Therapy, 93: 17-21.

Culp \& Romani (2006). Physical therapist examination, evaluation and intervention following the surgical reconstruction of a grade III acromioclavicular join separation. descriptive study. Physical thrapy.

Ellenbecker, Pluim, Vivier, \& Sniteman (2009) Common Injuries in Tennis Players: Exercises to Address Muscular Imbalances and Reduce Injury Risk. Strength and Conditioning Journal. 31(4).www.nsca-lift.org.

English, Hillier, Stiller \& Warden-Flood (2007). Circuit Class Therapy Versus Individual Physiotherapy Sessions During Inpatient Stroke Rehabilitation: A Controlled Trial. Archieves of Physical Medicine and Rehabilitation. Vol 88: 955-963.
Fernandez-Fernandez, J.; Ellenbecker, T.; Sanz-Rivas, D.; Ulbricht, A.; \& Ferrauti, A. (2013). Effects of a 6 week junior tennis conditioning program on service velocity. Journal of Sports Science and Medicine.12, 232-239.

Fitzgerald, G.; Piva, S.; Gil, A.; WIsnnelwski, S.;Oddis, Ch. \& Irragang, J. (2011). Agility and Perturbation Training Techniques in Exercise Therapy for Reducing Pain and Improving Function in People With Knee Osteoarthritis.A randomized Trial.Physical Therapy. 91:452-469. Recuperado de http//www.apta.org.

Fragala-Pinckham, M.; Jaley, S.; Rabin, J.; \& Kharasch, V. (2005). A Fitness Program for Children With Disabilities.Physical Therapy 85: 1182-1200.

Frank, C.; Kovesova, A.; \& Kolar, P. (2013).Clinical commentary dynamic neuromuscular stabilization \& sports rehabilitation. The International Journal of Sports Physical Therapy 8 (1)

Hale, Hertel \& Olmsted-Kramer (2007). The Effect of a 4-Week comprehensive Rehabilitation Program on Postural Control and Lower Extremity Function in Individuals With Chronic Ankle Instability.experimental study clinical trial. Journal of orthopedic sport physical therapy, Vol 37 Num 6.

Hernández, R.; Fernández, C.; \& Baptista, P. (2012). Metodología de la Investigación. Cuarta Edición. México McGraw Hill.

Inge E.P.M., Van Haren, Timmermant, H., Potting, C.M. Blijle, N., Vens, J., Slaal, B. \& Huis -Vander Sanden, M. W. (2013). Physical Exercise for Patients Undergoing Hematopoietic Stem Cell Transplantation: Systematic review and Meta-Analyses of Randomized Controlled Trials. Meta-Analyses of Randomized Controlled Trials. Phisycal Therapy, 93:512-528.

Jacobs, Lewis, Bolgla, Chistersen, Nitz, \& Uhl (2009). Electromyographic Analysis of Hip abductor exercises performed by a sample of Total Hip Arthroplasty patients. The Journal of Arthroplasty Vol. 24 (7).

Johansson, K.; Adolfsson, L.; \& Foldevi, M. (2005) Effects of acupunture versus ultrasound in patients with impingement syndrome: randomized clinical trial. Physical Therapy. 85:490-501.

Kemmler, Lauber, Weineck, Hensen, Kalender \& Engelke (2006). Benefits of 2 Years of Intense Exercise on Bone Density, Physical Fitness, and Blood Lipids in Early Postmenopausal Osteopenic Women. Descriptive study. Rescatado de www.archintermed.com

Liu, C. \& Latham, N. (2009). Progressive resistance strength training for improving physical function in older adults (Review). The Cochrane Collaboration. Published by John Wiley \& Sons, Ltd.

López Chicharro, J. (2001). Fisiología del Ejercicio. 2da Edición. Madrid, España. Editorial Médica Panamericana.

REV. COL. REH 2014 || Volumen 13 || Páginas 106 - 116 || 
López Miñaro, P. (s.f.). Fortalecimiento lumbo-abdominal y estabilidad de la columna vertebral.Universidad de Murcia. Recuperado de http:// digitum.um.es/xmlui/bitstream/10201/5245/1/fortalecimiento $\% 20$ de\%2ola\%2omusculatura\%2odel\%2otronco.pdf

Manterola, C.; y Zavando D. (2009). Cómo interpretar los "Niveles de Evidencia" en los diferentes escenarios clínicos. Revista Chilena de Cirugía. Vol 61 (6); p.582-595.

Mattacola, C. \& Dwyer, M. (2002). Rehabilitation of the Ankle After Acute Sprain or Chronic Instability.Journal of Athletic Training. 37(4): $413-429$.

Moser, M. (2009). Treatment for a 14-Year-Old Girl With Lyme Disease Using Therapeutic Exercise and Gait Training. Physical Therapy. 91:1412-1423. doi: 10.2522/ptj.20110020.

Myer, Ford, Palumbo \& Hewitt (2005). Neuromuscular training improves performance and lower-extremity biomechanics in female athletes. Experimental study clinical trial. Journal of strength and conditioning research, 19 (1) 51-60.

Nilsson, Jonsson \& Ekdahl (2009). Effects of a training program after surgically treated ankle fracture: a prospective randomized controlled trial. Recuperado de www.biomedcentral.com.

Page, Lamberth, Abadie, Boling, Collins \& Linton (1993). Posterior Rotator Cuff Strengthening Using Theraband ${ }^{\circledR}$ in a Functional Diagonal Pattern in Collegiate Baseball Pitchers; Clinical trial.Journal of athletic training, Vol 28 Number 4.

Piva, S.; Gil, A.; Almeida, G.; DIgioia, D.; Levison, T.; \& Fitzgerald, K. (2010). Balance Exercise Program Appears to Improve Function for Patients With Total Knee Arthroplasty: A Randomized Clinical Trial. Physical Therapy 90 (6).

Preisinger, Alacamlioglu, Pils, Bosina, Metka, Schneider \& Ernst (2011). Exercise therapy for osteoporosis: results of a randomized controlled trial. Canadian Medical Association.

Schmitt, LC.; Schmitt, LA.; \& Rudolph, K. (2004) Management of a patient with a forearm fracture and median nerve Injury. Journal of Orthopaedic\& Sports Physical Therapy.34(2).

Smith, Docherty, Simon, Klossner \& Schrader (2012). Ankle strength and force sense after a progressive -6 week strngth- training program in people with functional ankle inestability; Randomized controlled clinical trial. Journal of athletic training, (3) 282-288.

Stevenson, M.; Warpeha, J.; Dietz, C.; Giveans, R. \& Erdman, A. (2010) Acute effects of elastic bands during the free-weight barbell back squat exercise on velocity, power, and force production. Journal of strength and conditioning research / National Strength \& Conditioning Association. 24 (11):2944-54
Swank, A.; Kachelman, J.; Bibeau, W.; Quesada, P.; Nyland, J.; Malkani, A. \& Toop, R. (2011). Prehabilitation before total knee arthroplasty increases strength and function In older adults with severe osteoarthritis. Journal of Strength and Conditioning Research. 25(2)/318-325.

Szturm, T.; Betker, AL.; Moussavi, Z.; Desai, A.; \& Goodman, V. (2011). Effects of an Interactive Computer Game Exercise Regimen on Balance Impairment in Frail Community-Dwelling Older Adults: A Randomized Controlled Trial. Physical Therapy; 91:1449-1462. doi: 10.2522/ ptj.20090205.

The Hygenic Corporation (2006). Bandas \& Ligas de Resistencia, Manual de Instrucciones. Volúmen 4. recuperado de http://www.thera-bandacademy.com/elements/clients/docs/Thera-BandInstructionSpanish_201108DD_054742.pdf

Theraband, Systems of progressive exercise (sf). (http://www.thera-band. sporty.pl/historia.shtml.

Tonley, J.; Yun, S.; Kochevar, R.; Dye, J.; Farrokhi, S.; \& Powers, Ch. (2010). Treatment of an Individual With Piriformis Syndrome Focusing on Hip Muscle Strengthening and Movement Reeducation: A Case Report. Journal of Orthopaedic\& Sports Physical Therapy 40(2).

Topp, R.; Pague, P.; Swank, A.; Quesada, P.; Nyland, J.; \& Malkani, A. (2009). Improve function before knee replacement surgery. Functional $U$, excercise and activity for healthy aging. Marzo - Abril.

Universidad de Antioquia (sf). Guía curricular para la educación Física. Las capacidades Fisiocomotrices. Recuperado el 25 de noviembre de 2013, de http://docencia.udea.edu.co/edufisica/guiacurricular/Fisicomotrices.pdf.

Unsgaard-Tøndel M, Fladmark AM, Salvesen $\varnothing$ \& Vasseljen O (2014). Motor Control Excercises, sling excercises and general excercises for patientes with chronic low back pain; Randomized controlled clinical trial. Phisycal therapy, 90:1426-1440.

Van Ettekoven, H. \& Lucas, C. (2006). Efficacy of physiotherapy including a craniocervical training programme for tension-type headache; a randomized clinical trial. Cephalalgia. 26: 983-991.

Wang, Ch.; McClure, Ph. Pratt, N.; \& Nobilini, R. (1999). Stretching and Strengthening Exercises: Their Effect on Three-Dimensional Scapular Kinematics. Archives of Physical Medicine and Rehabilitation 80.

Wilcox III, R.; Arslanian, L.; \& Millett, P. (2005).Rehabilitation following total shoulder arthroplasty.descriptive study. Journal of orthopedic and sports physical therapy, vol 35, num 12.C8

Witt, Talbott \& Kotowski (2011). Electromyographic activity of scapular muscles during diagonal patterns using elastic resistance and free weights. Clinical trial. The intenational journal of sports physical therapy, Vol 6, number 4 . 